\title{
Extraventricular Choroid Plexus Carcinoma with Spinal Metastasis: A Case Report
}

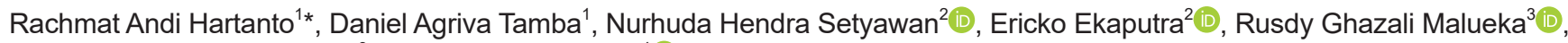
Indra Sari Kusuma Harahap ${ }^{3}$, Ery Kus Dwianingsih ${ }^{4}$ (D)

${ }^{1}$ Department of Surgery, Division of Neurosurgery, Faculty of Medicine, Public Health and Nursing, Universitas Gadjah Mada, Dr. Sardjito General Hospital, Yogyakarta, Indonesia; ${ }^{2}$ Department of Radiology, Faculty of Medicine, Public Health and Nursing, Universitas Gadjah Mada,Dr. Sardjito General Hospital, Yogyakarta, Indonesia; ${ }^{3}$ Department of Neurology, Faculty of Medicine, Public Health and Nursing, Universitas Gadjah Mada,Dr. Sardjito General Hospital, Yogyakarta, Indonesia; ${ }^{4}$ Department of Anatomical Pathology, Faculty of Medicine, Public Health and Nursing, Universitas Gadjah Mada,Dr. Sardjito General Hospital, Yogyakarta, Indonesia

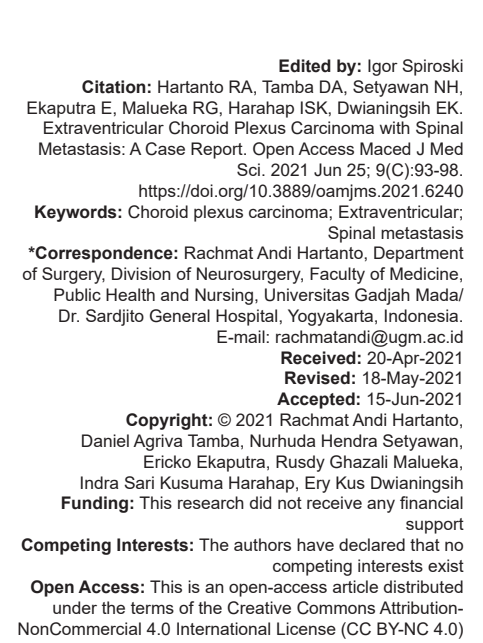

Introduction

Choroid plexus carcinoma (CPC) is a rare intra-cranial tumor originating from the choroid plexus which belongs to the choroid plexus tumor (CPT) group [1]. This tumor is primarily a childhood tumor, with a median age of 3-3.5 years [2], [3]. All CPT variants are mostly intra-ventricular tumor, where about half of them are inside the lateral ventricle [1]. Nevertheless, several reports describe the extraventricular origin of CPC [4], [5]. Concerning metastatic capability, CPC has a higher tendency to metastasize than choroid plexus papilloma (CPP) [3]. To the best of our knowledge, this is the first article to describe an extraventricular CPC with spinal cord metastasis

\begin{abstract}
BACKGROUND: We report a case of extraventricular choroid plexus carcinoma (CPC) accompanied by spinal

reAs: A 15-year-old female patient presented with chronic progressive headache and blurry vision. Magnetic and the histopathological examination revealed the diagnosis of CPC, confirmed with immunohistochemical (IHC) mass in the left frontal lobe. Spinal MRI showed drop metastasis to the spine, accompanied by leptomeningeal

CONCLUSION: This case demonstrated the importance of IHC and spinal MRI in the management of CPC. This case report may provide more insight into extraventricular $\mathrm{CPC}$ cases, which is important for the adequate management
\end{abstract} of patients with CPC in the future.

\section{Case Presentation}

A 15-year-old teenager presented with chronic progressive, mild-to-moderate-intensity headache, blurry vision, and pain around her left orbit, 1 year before hospital admission. Other complaints such as seizure, memory deficit, weakness on the extremities, or behavioral changes were not present. History of medical conditions or malignancies in her family was absent. After visiting an ophthalmologist, she was diagnosed with an acute papillitis and hospitalized for 3 days. A magnetic resonance imaging (MRI) of her brain was performed in June 2019 and showed an intra-axial mass on the left frontal lobe with a mixture of cystic and solid components (Figure 1). We initially suggested that the patient had developed a high-grade 

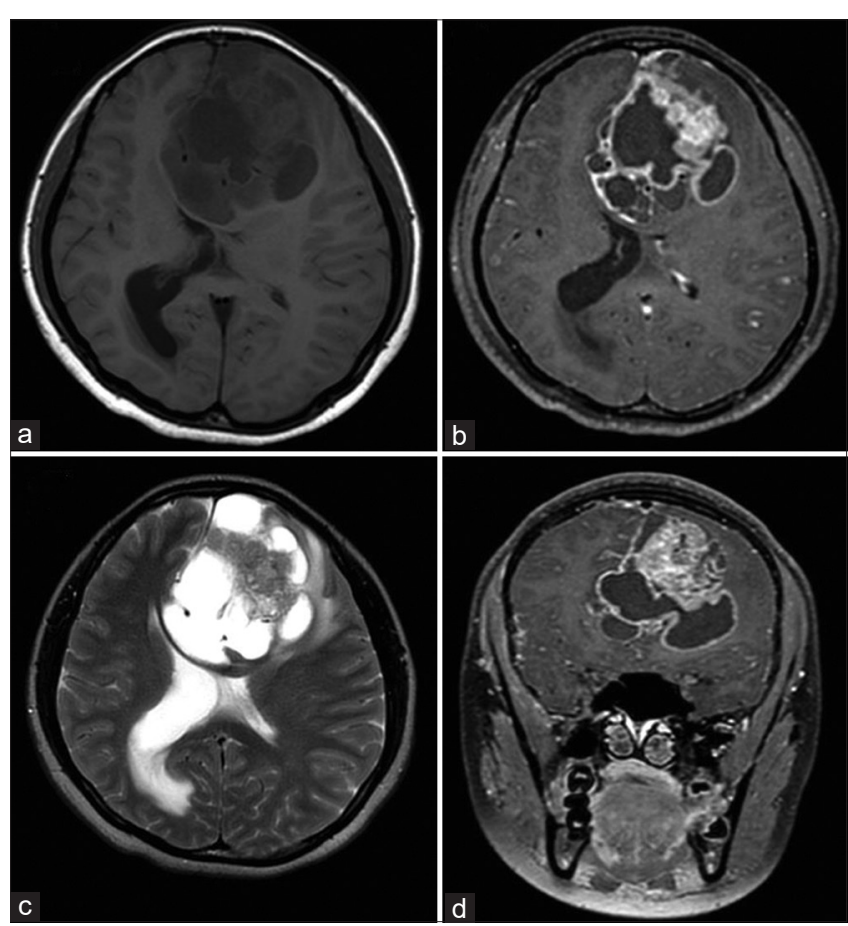

Figure 1: Pre-operative contrast-enhanced brain magnetic resonance imaging (a-c, axial views; $d$, coronal view). (a) Iso- and hypointense tumor in pre-contrast T1-weigthed image suggesting mixture of solid and cystic components. (b) Contrast-enhanced T1-weighted image showing enhancement on the tumor wall and the solid component. (c) T2-weighted image showing a hyperintense predominantly cystic lesion with moderate perifocal edema and resultant obstructive hydrocephalus. (d) Coronal contrast-enhanced T1-weighted image showing the left cingulate gyrus of tumor herniated to the right hemisphere

glioma. The patient then consented for the craniotomy procedure in July 2019. An extended left uni-coronal incision and left frontal craniotomy was performed in Dr. Sardjito General Hospital. The tumor was removed using a left frontal approach. A well-demarcated tumor, with rubbery consistency and some cystic components, was observed and sent for pathology examination. After the surgery, the patient awoke with some cognitive dysfunctions.

The pathology report described a tumor that infiltrated the surrounding normal brain tissue in papillary and solid growth fashion, partly forming a pseudo-rosettes pattern around the vascular (Figure 2), suggesting an anaplastic ependymoma (WHO Grade III) with differential diagnosis of CPC. Furthermore, immunohistochemical (IHC) analysis of cytokeratin (CK), S100, epithelial membrane antigen (EMA), and glial fibrillary acidic protein (GFAP), was performed (Figure 3) and confirmed the diagnosis of CPC since the primary tumor site in other organs was not detected in patient. Post-operative imaging revealed a parenchymal defect on the left frontal lobe with subacute intra-tumoral hematoma. The residual tumor could not be seen clearly on the left frontal lobe. The patient then received a fractionated proton beam radiotherapy to the tumor bed with a total dose of $60 \mathrm{~Gy}$ over 30 treatments.

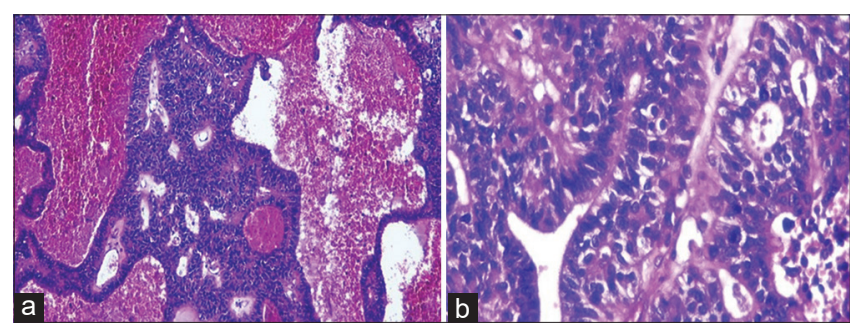

Figure 2: (a) Resected tumor stained with Hematoxylin Eosin (HE) showed papillary growth fashion, partly forming pseudorosettes around the vascular, with wide area of bleeding, infiltrating surrounding tissue (100× magnification). (b) Tumor cells were cuboid to columnar, atypical, and hyperchromatic (400× magnification)

In February 2020, the patient developed lower back pain, followed by numbness on the perineal region, and weakness on the lower extremities. A whole spine MRI with contrast revealed multiple well-defined solid intra-dural extramedullary lesions in various spinal regions (Figure 4). A follow-up contrast-enhanced brain MRI was performed and exhibited new multiple pathological lesions within the left frontal lobe, with a well-defined border. There were also multiple lesions located on the bilateral temporal lobes and bilateral cerebellum and multiple meningeal enhancements (Figure 4), suggesting a recurrent lesion of the CPC. Decompressive laminectomy for tumor debulking was performed in June 2020. The histopathological analysis of the spinal mass suggested the diagnosis: Metastasis of CPC (Figure 5), supported by $\mathrm{IHC}$ analysis that revealed a strong positivity of CK, with focal positivity of S100 in the tumor cells. Meanwhile, GFAP and EMA were negatively expressed (Figure 5). These results confirmed the diagnosis of drop metastasis of CPC from the left frontal lobe to the spine. Other radiotherapy treatment for the cranial and spinal lesions was planned, however, unfortunately, her condition worsened, and after five fractions of radiotherapy, she did not survive further treatments.

\section{Discussion}

CPC, a rare tumor, usually occurs within the ventricular system, with the lateral ventricle being the most common site for this tumor to grow [1], [2]. We presented a very rare case, where the patient developed an extraventricular CPC, which mainly resided in the left frontal lobe. The exact oncogenesis of this extraventricular tumor is still unclear. The ectopic choroid plexus was a possible explanation of extraventricular CPC [6]. Another potential mechanism is that these tumors arose from ependymal tissue that, in the later phase of brain development, was separated from the entire ependyma [7]. 


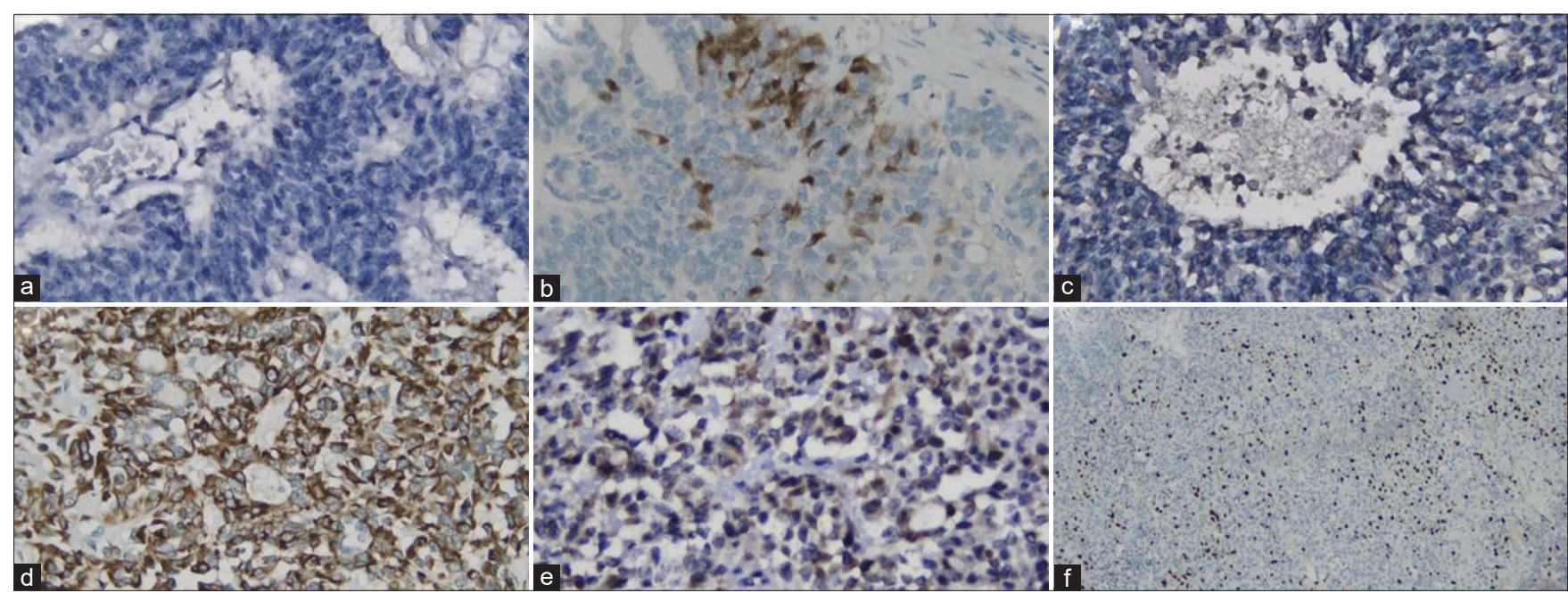

Figure 3: The immunohistochemistry result of the brain tumor showed no expression of glial fibrillary acidic protein (GFAP) (a), locally positive immunostaining of $S 100$ (b), and no immunoreactivity of EMA in tumor cells membrane (c). cytokeratin (CK), an epithelial cell marker, was strongly stained in cytoplasm of tumor cells (d), meanwhile P53 mutant was strongly stained in tumor cells' nuclei (e). Ki67, a proliferation marker of the cells, was strongly stained in the nuclei of tumor cells, showing $40 \%$ of proliferation index ( $f$ )

The pathology of CPC shows an increased cell density, a high mitotic index, nuclear pleomorphism, and necrotic areas [6]. Moreover, $\mathrm{IHC}$ analysis for CPC exhibits $\mathrm{CK}$ and mutant P-53 immunopositivity, and negative immunostaining for GFAP and EMA [8]. Initially, we diagnosed our case as an extraventricular anaplastic ependymoma, with typical ependymal appearances, focal perivascular pseudo-rosettes, and increased cellularity on the histopathology examination. In this case, the $\mathrm{IHC}$ has an essential role in differentiating both diseases, where ependymoma is positively stained for GFAP [9]. We then could rule out ependymoma as the diagnosis.

Among all types of CPT, CPC has the highest recurrence rate. A report from one center described that among all CPT cases treated, CPC cases were 20 times more likely to recur [10]. In that study, recurrence occurred in four of seven patients. In another study investigating 36 CPT cases (21 CPP or CPP, 11 atypical CPP, and 4 CPC), two out of the four CPC patients developed tumor recurrence [11]. One recurrence case of $C P C$ was treated non-surgically using chemotherapy and gamma-knife. In that study, three cases, including one CPC case, underwent subtotal resections. Tumor recurrence was reported to occur in two out of five cases of extraventricular tumors [5], [12]. In these cases, the recurrence occurred between 4.5 months and 5 years after treatment completion.

Metastatic lesion from CPC had been described in several articles, where drop metastasis and leptomeningeal involvement were the most frequent metastatic lesions mentioned in these studies [13], [14], [15]. Our patient developed both drop metastasis and leptomeningeal seeding (LS). There are several ways for the tumor to spread to cerebrospinal fluid (CSF), and tumor invasion from choroid plexus into CSF is one possible explanation. Other means include hematogenous spread through vessels in subarachnoid space, direct invasion of the tumor from the parenchyma, or retrograde perineurial spread to the subarachnoid space through the cranial nerves. In addition, several authors argued that surgical resection increases the likelihood of LS occurrence [16], [17]. However, in our case, we could not determine the exact period of LS emergence due to the absence of postcraniotomy whole-spine MRI.

Furthermore, until now, there has been no clear guideline to manage tumors of the choroid plexus. Several studies emphasized the importance of gross total resection (GTR) in reducing the recurrence rate in CPT [1], [3], [10], because it was considered the most significant factor to determine the patient's overall survival (OS) [2], [3]. On the other hand, a 20-year single institutional study stated that GTR might reduce the post-operative symptoms related to the increased intracranial pressure, yet could not improve the survival of patients with CPT, especially the CPC group [10]. Therefore, it was reasonable to highlight the need for adjuvant therapy. In one study, the 5-year projected survival rate in a pediatric group of patients who were $>3$ years old was higher than the group that did not undergo radiotherapy ( $68 \%$ vs. $16 \%$, respectively) [18]. Moreover, the role of chemotherapy in patients with CPC also appears to be promising. In a study, the prognosis of CPC patients with subtotal resection or in the patients with no history of radiation was significantly improved after chemotherapy [19]. Another systematic review on the effect of adjuvant in patients with CPC found that chemotherapy, both in the combination with radiotherapy group and in the chemotherapy alone group, significantly improved the OS [20]. 

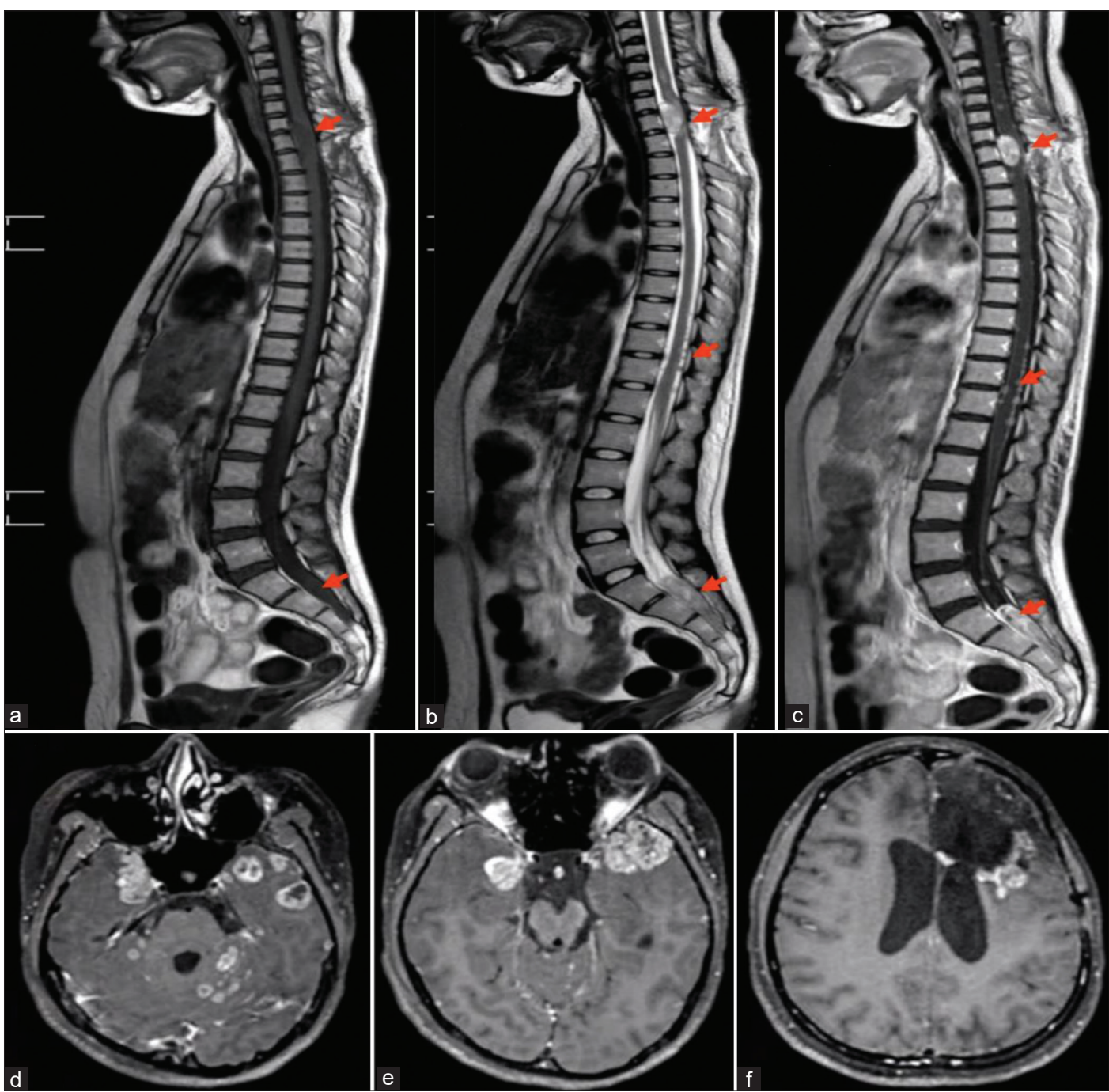

Figure 4: Follow-up contrast-enhanced whole spine and brain magnetic resonance imaging (MRI) following gross total resection and radiation, (a-c) Sagittal T1-weighted image, T2-weighted, and contrast-enhanced T1-weighted image, respectively; (d-f) Multi-level axial brain MRI with contrastenhanced T1-weighted image. Red arrow indicates multiple intensely-enhancing solid intra-dural extramedullary lesions in lower cervical, thoracic, and sacral spine which caused severe compression of spinal canal and medulla. Brain MRI revealed multiple solid-cystic intensely-enhancing new lesions in prior tumor location, temporal lobe, and also focal meningeal enhancements which suggested recurrent lesion and meningeal spread

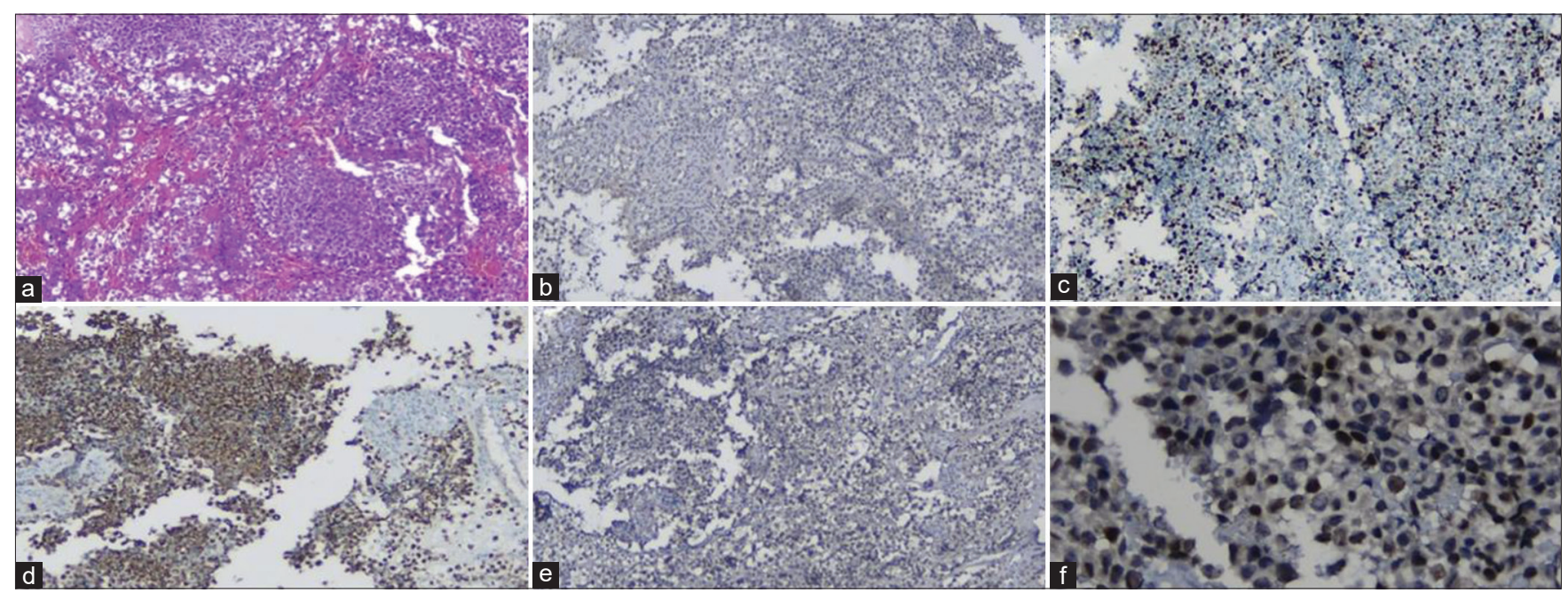

Figure 5: The morphology features and immunohistochemistry result of resected spinal mass. (a) Using Hematoxylin Eosin staining, tumor cells were in solid pattern growth, consisted of atypical cells with clear cytoplasm resembling clear cell type of ependymoma. Due to the previous history of choroid plexus carcinoma (CPC) in brain parenchyma, differential diagnosis of drop metastasis of CPC was also made. (b) Immunohistochemistry staining showed negative staining of glial fibrillary acidic protein. (c) Ki67 immunostaining showed $60 \%$ of proliferation index. (d) Cytokeratin was strongly stained in cytoplasm of tumor cells, meanwhile (e) epithelial membrane antigen was negatively stained. (f) P53 immunostaining of mutant P53 was highly expressed in the nucleus of tumor cells 


\section{Conclusions}

ThisisacaseofCPClocatedintheextraventricular region, manifested with metastatic lesions to the spine. Radiology and immunohistochemistry play an essential role in establishing the diagnosis of CPC. In addition, post-operative spine MRI is important and must be done as soon as possible to exclude the possibilities of spinal metastasis, which might complicate the management. Moreover, a guideline that describes the management of CPC, covering every aspect of therapy such as surgery, radiation therapy, and chemotherapy, is undoubtedly needed to reduce the uncertainty in the management of CPC. In the future, more high-quality CPC-related studies are necessary to help to improve our knowledge regarding this type of tumor.

\section{Informed Consent}

The patient was deceased at the time of this writing. Written informed consent was obtained from the patient's family member for publication of this case report and accompanying images. A copy of the written consent is available for review by the Editor-in-Chief of this journal on request.

\section{Acknowledgments}

We thank Hana Amatillah for useful discussions during this manuscript drafting.

\section{References}

1. Gupta N. Choroid plexus tumors. In: Pediatric CNS Tumors. Berlin, Germany: Springer International Publishing; 2017. p. 187-98. https://doi.org/10.1007/978-3-319-30789-3_9

2. Cannon DM, Mohindra P, Gondi V, Kruser TJ, Kozak KR. Choroid plexus tumor epidemiology and outcomes: Implications for surgical and radiotherapeutic management. J Neurooncol. 2015;121(1):151-7. https://doi.org/10.1007/s11060-014-1616-x PMid:25270349

3. Wolff JE, Sajedi M, Brant R, Coppes MJ, Egeler RM. Choroid plexus tumours. Br J Cancer. 2002;87(10):1086-91. https://doi. org/10.1038/sj.bjc.6600609

PMid:12402146

4. Stevens EA, Stanton CA, Nichols K, Ellis TL. Rare intraparenchymal choroid plexus carcinoma resembling atypical teratoid/rhabdoid tumor diagnosed by immunostaining for INI1 protein. J Neurosurg Pediatr. 2009;4(4):368-71. https://doi. org/10.3171/2009.5.peds0955

\section{PMid:19795969}

5. Carter AB, Price DL, Tucci KA, Lewis GK, Mewborne J, Singh HK. Choroid plexus carcinoma presenting as an intraparenchymal mass. J Neurosurg. 2001;95(6):1040-4. https://doi.org/10.3171/ jns.2001.95.6.1040

\section{PMid: 11765820}

6. Lozier AP, Arbaje YM, Scheithauer BW. Supratentorial extraventricular choroid plexus carcinoma in an adult: Case report. Neurosurgery. 2009;65(4):E816-7. https://doi. org/10.1227/01.neu.0000348291.48810.c2

PMid:19834361

7. Greene RC. Extraventricular and intra-cerebellar papilloma of the choroid plexus. J Neuropathol Exp Neurol. 1951;10(2):204-7. https://doi. org/10.1097/00005072-195104000-00007

PMid: 14824975

8. Gopal P, Parker JR, Debski R, Parker JC. Choroid plexus carcinoma. Arch Pathol Lab Med. 2008;132(8):1350-4. https:// doi.org/10.5858/2008-132-1350-cpc

PMid: 18684041

9. Sun S, Wang J, Zhu M, Beejadhursing R, Gao P, Zhang X, et al. Clinical, radiological, and histological features and treatment outcomes of supratentorial extraventricular ependymoma: 14casesfromasinglecenter.JNeurosurg.2018;128(5):1396-402. https://doi.org/10.3171/2017.1.jns161422 PMid:28686116

10. Bettegowda C, Adogwa O, Mehta V, Chaichana KL, Weingart J, Carson BS, et al. Treatment of choroid plexus tumors: A 20-year single institutional experience. J Neurosurg Pediatr. 2012;10(5):398-405. https://doi.org/10.3171/2012.8.peds12132 PMid:22938081

11. Hosmann A, Hinker F, Dorfer C, Slavc I, Haberler C, Dieckmann K, et al. Management of choroid plexus tumors-an institutional experience. Acta Neurochir (Wien). 2019;161(4):745-54. https:// doi.org/10.1007/s00701-019-03832-55

PMid:30783805

12. Samuel T, Parikh J, Sharma S, Giller C, Sterling K, Kapoor S, et al. Recurrent adult choroid plexus carcinoma treated with high-dose chemotherapy and syngeneic stem cell (bone marrow) transplant. J Neurol Surg Part A Cent Eur Neurosurg. 2013;74(1):e149-54. https://doi.org/10.1055/s-0032-1333419 PMid:23427033

13. Sawaishi Y, Yano T, Yoshida Y, Ito Y, Mizoi K, Hirayama A, et al Choroid plexus carcinoma presented with spinal dysfunction caused by a drop metastasis: A case report. J Neurooncol. 2003;63(1):75-9.

PMid: 12814258

14. Baksh BS, Sinha N, Salehi A, Han RH, Miller BA, Dahiya S, et al. Widely metastatic choroid plexus carcinoma associated with novel TP53 somatic mutation. World Neurosurg. 2018;119:233-6. https://doi.org/10.1016/j.wneu.2018.07.284 PMid:30099178

15. Taylor MB, Jackson RW, Hughes DG, Wright NB. Magnetic resonance imaging in the diagnosis and management of choroid plexus carcinoma in children. Pediatr Radiol. 2001;31(9):624-30. https://doi.org/10.1007/s002470100511 PMid:11512001

16. Van Der Ree TC, Dippel DW, Avezaat CJ, Sillevis Smitt PA, Vecht CJ, Van Den Bent MJ. Leptomeningeal metastasis after surgical resection of brain metastases. J Neurol Neurosurg Psychiatry. 1999;66(2):225-7. https://doi.org/10.1136/ jnnp.66.2.225

PMid: 10071105

17. Norris LK, Grossman SA, Olivi A. Neoplastic meningitis following 
surgical resection of isolated cerebellar metastasis: A potentially preventable complication. J Neurooncol. 1997;32(3):215-23.

\section{PMid:9049883}

18. Wolff JE, Sajedi M, Coppes MJ, Anderson RA, Egeler RM. Radiation therapy and survival in choroid plexus carcinoma. Lancet. 1999;353(9170):2126. https://doi.org/10.1016/ s0140-6736(99)01744-4

PMid:10382700

19. Wrede B, Liu P, Wolff JE. Chemotherapy improves the survival of patients with choroid plexus carcinoma:
A meta-analysis of individual cases with choroid plexus tumors. J Neurooncol. 2007;85(3):345-51. https://doi.org/10.1007/ s11060-007-9428-x

PMid: 17576522

20. Sun MZ, Ivan ME, Oh MC, Delance AR, Clark AJ, Safaee M, et al. Effects of adjuvant chemotherapy and radiation on overall survival in children with choroid plexus carcinoma. J Neurooncol. 2014;120(2):353-60. https://doi.org/10.1007/ s11060-014-1559-2

PMid:25106865 